\title{
Caracterización de las poblaciones de caballitos de mar en diferentes zonas de la costa norte de las regiones occidental y central de Cuba
}

\section{Characterization of seahorse populations in different areas in the north coast in the western and central regions of $\mathrm{Cuba}$}

\author{
Lourdes Pastor Gutiérrez ${ }^{1 *}$, Daril de la Nuez Hernández ${ }^{1}$, Raúl Igor Corrada Wong ${ }^{1}$, Yuliet \\ Piloto Cubero ${ }^{I}$ y Alejandro Pérez Angulo ${ }^{l}$
}

\begin{abstract}
RESUMEN
Los caballitos de mar son peces ornamentales de gran interés para las exhibiciones en el Acuario Nacional de Cuba, por lo que se propuso valorar el estado actual de las poblaciones naturales de las especies Hippocampus reidi (Ginsburg, 1933) e Hippocampus erectus (Perry, 1810) en diferentes zonas de la costa norte de las regiones occidental y central de la isla. Se llevaron a cabo censos visuales mediante buceo libre, utilizando el método de transectos lineales, entre marzo del 2007 y marzo del 2010. Para la comparación de medias se aplicaron las pruebas de Kruskal Wallis y U de Mann-Whitney. La mayor densidad se encontró en las estructuras artificiales de las estaciones de Barracuda y Caleta, probablemente debido a la gran cantidad de sitios de anclaje presentes y la eutrofización. No se reportaron diferencias en esta variable entre las épocas de lluvia y seca para ninguna de las dos especies. H. reidi fue la especie más abundante y la de mayor distribución. Aunque la densidad de individuos de hipocampos suele ser baja, es posible que la marcada escasez de ejemplares de H. erectus en este caso se deba al método de muestreo (profundidad máxima de 2 metros). Los machos de ambas especies fueron mayores que las hembras. $H$. reidi se reprodujo todo el año y la proporción sexual registrada fue muy similar a la proporción 1:1, mientras que en $H$. erectus esta variable fue diferente a la esperada. Las macroalgas del género Caulerpa fueron los sustratos más utilizados por ambas especies.
\end{abstract}

Palabras claves: Hippocampus reidi, Hippocampus erectus, caballito de mar, densidad, Cuba.

\begin{abstract}
Seahorses are ornamental fish of great interest for exhibits in the Cuban National Aquarium; therefore, the current state of natural populations of the species Hippocampus reidi (Ginsburg, 1933) and Hippocampus erectus (Perry, 1810) was assessed in different areas in the north coast of western and central regions in Cuba. Visual censuses were conducted by free diving, using the linear transects method, between March 2007 and March 2010. Means were compared using Kruskal-Wallis and Mann-Whitney U tests. The largest densities were found in the artificial structures in the Barracuda and Caleta stations, probably due to the large number of anchor sites and eutrophication. No differences were recorded in this variable for either species between the rainy and dry seasons. H. reidi was the most abundant species and the most widely distributed. Although the density of seahorse individuals is generally low, the marked shortage of $H$. erectus specimens may have been due to the sampling method (maximum depth 2 meters). There were more males than females in both species. H. reidi reproduced all year long and the sexual ratio recorded was very similar to 1:1 ratio, while, in $H$. erectus, this variable was different than expected. Macroalgae of the genus Caulerpa was the substrate most widely used by both species.
\end{abstract}

Keywords: Hippocampus reidi, Hippocampus erectus, seahorse, density, Cuba.

1 Acuario Nacional de Cuba. Ministerio de Ciencia, Tecnología y Medio Ambiente. Ave. 1ra. No. 6024. Miramar. Playa. La Habana CP 11300 Cuba. lourdesp@acuarionacional.cu*

Recibido: 1 de agosto de 2015

Corregido: 9 de noviembre de 2016

Aceptado: 16 de noviembre de 2016

DOI: http://dx.doi.org/10.15359/revmar.9-1.2 


\section{INTRODUCCIÓN}

Los caballitos de mar (género Hippocampus) son peces muy peculiares por las características morfológicas que exhiben. Debido a su valor en la medicina tradicional asiática y como especie ornamental, son víctimas frecuentes del comercio internacional (Vincent, 1995; Loh et al. 2016). Todo ello, junto con la degradación de sus hábitats, la captura indiscriminada para artesanías, el uso de artes de pesca poco selectivas y los cambios climáticos han provocado una declinación de sus poblaciones (Yasué et al. 2015; Harasti, 2016). Por esta razón, desde el año 2004 todas las especies del género Hippocampus están incluidas en el Apéndice II de la Convención sobre el Comercio Internacional de Especies Amenazadas de Fauna y Flora Silvestres (CITES, 2004) y en la Lista Roja de especies amenazadas (UICN, 2009).

De las 41 especies de caballitos de mar reconocidas como válidas por Lourie et al. (2016), en Cuba solo se han encontrado dos especies: Hippocampus erectus (Perry, 1810) e Hippocampus reidi (Ginsburg, 1933), las cuales desde el año 2011 están incluidas en el Apéndice II de la Resolución No. 160/2011 sobre Regulaciones para el control y la protección de especies de especial significación para la diversidad biológica en Cuba (Gaceta Oficial de la República de Cuba, 2011).

A partir de las consideraciones anteriores, el Acuario Nacional ha implementado un programa de investigaciones para determinar la abundancia de las poblaciones de caballitos de mar (Pastor et al. 2011), la ecología de estas especies (de la Nuez et al. 2016) y las técnicas para su reproducción en cautiverio con miras a recuperar sus poblaciones. En este contexto, el presente trabajo tiene como objetivo caracterizar las poblaciones de caballitos de mar en diferentes zonas de la costa norte de las regiones central y occidental de Cuba, determinando aspectos importantes de su ecología como: densidad, proporción sexual, período reproductivo, composición de los grupos y principales sustratos de apoyo, que nos ayuden a trazar estrategias integrales de manejo y protección para estas especies.

\section{MATERIALES Y MÉTODOS}

Se estudiaron 11 estaciones ubicadas en la costa norte de las regiones central y occidental de Cuba (Fig. 1): Las Cuarenta (Bahía de Cabaña), El Casino (Santa Fe), Santa Ana (Bajo), Barracuda y Caleta (Varadero), Refugio, Cerco, Cayo Francés y Marina Periquillo (Caibarién), Playa Flamenco y Canalizo de los Perros (Cayo Coco).

Se realizaron censos visuales entre las 8 y las 10 de la mañana, mediante buceo en apnea, a una profundidad que osciló entre 1 y $2 \mathrm{~m}$, que era la máxima en los diferentes biotopos estudiados. Se hicieron transectos de $20 \mathrm{~m}$ de largo por $1 \mathrm{~m}$ de ancho como modificación de la metodología propuesta por Curtis et al. (2004). El número de transectos varió por estaciones de acuerdo con 

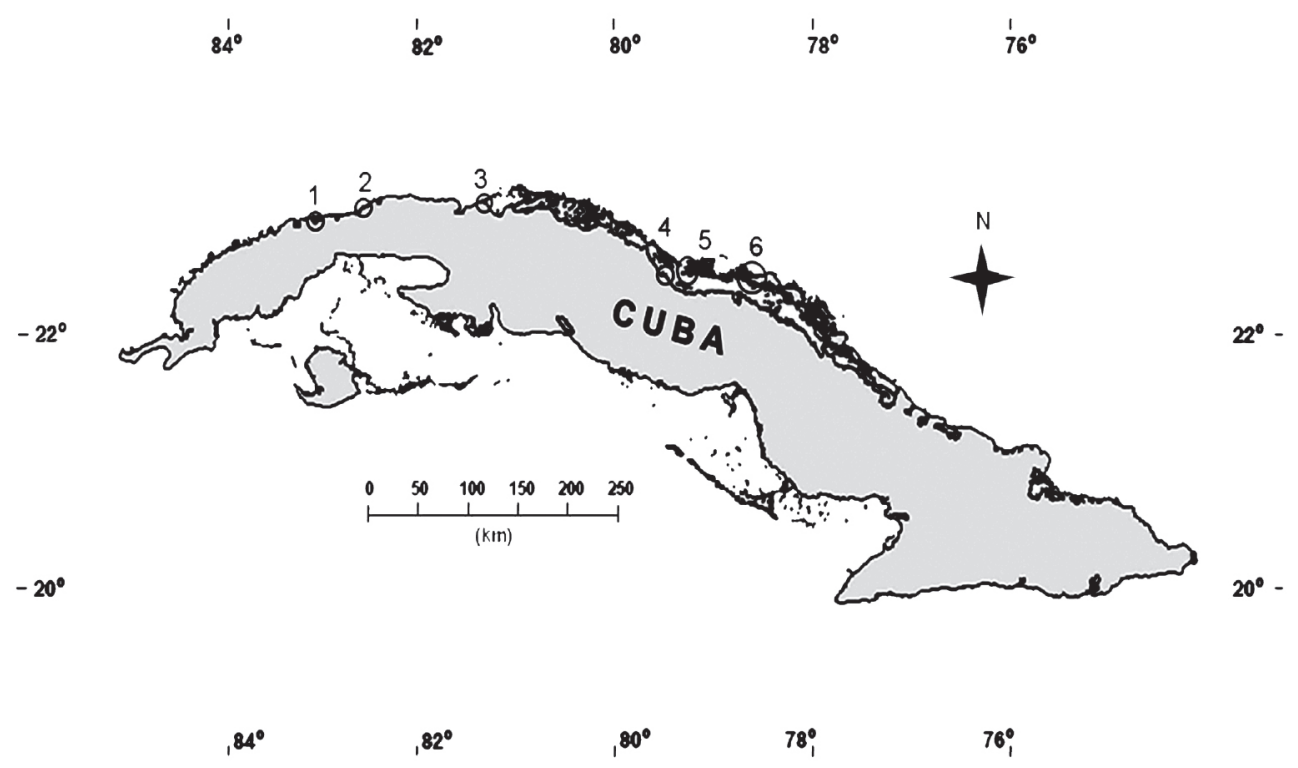

Fig. 1. Ubicación de las estaciones en la costa norte de las regiones occidental y central de Cuba. 1: Las Cuarenta; 2: Santa Ana y El Casino; 3: Barracuda y Caleta; 4: Refugio; 5: Cerco, Cayo Francés y Marina Periquillo; 6: Playa Flamenco y Canalizo de los Perros Fig. 1. Location of stations in the north coast in the western and central regions of Cuba. 1: Las Cuarenta; 2: Santa Ana and El Casino; 3: Barracuda and Caleta; 4: Refugio; 5: Cerco, Cayo Francés and Marina Periquillo; 6: Playa Flamenco and Canalizo de los Perros

las características geográficas de cada localidad (Cuadro 1). Los muestreos se efectuaron entre marzo del 2007 y marzo del 2010. Se realizaron tres muestreos en época de lluvia y tres en época de seca, con excepción de la estación de Barracuda, donde se realizaron cuatro en cada época.

A los individuos recolectados se les determinó in situ: la especie según Lourie et al. (1999), el sexo, la gravidez o no del macho y el largo total (LT) desde el surco medio de la corona hasta el extremo de la cola, con una regla graduada en centímetros (error $0,1 \mathrm{~cm}$ ). Todos los individuos menores de $6 \mathrm{~cm}$ de longitud total
(LT) se consideraron indiferenciados. Además, se anotaron el color y el tamaño del grupo, igualmente se identificó el tipo de sustrato en que se encontró cada individuo.

Se determinaron algunos parámetros físicoquimicos del agua como temperatura y salinidad. La temperatura se midió con un termómetro (in situ), mientras que la salinidad se determinó con un refractómetro de 1 unidad estándar de salinidad en el laboratorio.

Para el análisis estadístico de los datos solo se tomaron en cuenta aquellas estaciones donde se vieron más de tres individuos, por lo que no se incluyen las zonas de Canalizo de los Perros 
Cuadro 1. Datos poblacionales de $H$. reidi por estaciones. N: número de individuos; n: número de transectos; M.H: proporción sexual; Media \pm intervalo de confianza (rango); LT: Largo Total y entre paréntesis aparece el rango, H: hembra, M: macho; U: prueba de Mann-Whitney para el LT y para la densidad en época de lluvia y seca

Table 1. Population data for $H$. reidi per station. N: number of individuals; n: number of transects; M.H: sex ratio (male and female), mean \pm confidence interval; LT: Total length (range between parentheses), $\mathrm{H}$ : female, $\mathrm{M}$ : male U: Mann-Whitney test for TL and for density in rainy and dry seasons

\begin{tabular}{|c|c|c|c|c|c|c|c|c|}
\hline Estación & $\mathbf{N}(\mathbf{n})$ & M:H & $\begin{array}{l}\text { Densidad media } \\
\text { ind } / \mathbf{m}^{2}\end{array}$ & $\begin{array}{l}\text { Prueba U: } \\
\text { seca/lluvia }\end{array}$ & $\begin{array}{c}\text { LT } \\
(\mathrm{cm})\end{array}$ & $\begin{array}{l}\text { LT } \\
\text { (M) }\end{array}$ & $\begin{array}{l}\text { LT } \\
\text { (H) }\end{array}$ & $\begin{array}{c}\text { Prueba } \\
\text { U: } \\
\text { MxH } \\
\text { (LT) }\end{array}$ \\
\hline \multirow[t]{2}{*}{$\begin{array}{l}\text { Las } \\
\text { Cuarenta } \\
\text { (B. Cabaña) }\end{array}$} & \multirow[t]{2}{*}{$20(128)$} & $0.9: 1$ & $0.0078 \pm 0.0018$ & \multirow[t]{2}{*}{$\begin{array}{l}\left(\mathrm{U}_{64.64} 1787\right. \\
P=0.04)\end{array}$} & 7.25 & 8.4 & 7.7 & $\mathrm{U}=8$ \\
\hline & & $\mathrm{X}^{2}=0.00 P<0.9$ & $(0-0.0156 \pm 0.0075)$ & & $(4-11)$ & $(6-11)$ & $(6.2-9.8)$ & $P<0.04$ \\
\hline Santa Ana & $20(60)$ & $\begin{array}{l}1.8: 1 \\
\mathrm{X}^{2}=0.94 P<0.5\end{array}$ & $\begin{array}{l}0.0175 \pm 0.0031 \\
(0.01-0.023 \pm 0.0082)\end{array}$ & $\begin{array}{l}\left(\mathrm{U}_{30.30}=435\right. \\
P=0.79)\end{array}$ & $\begin{array}{l}11.5 \\
(4.9-15.2)\end{array}$ & $\begin{array}{l}13.7 \\
(12-15.2)\end{array}$ & $\begin{array}{l}10.4 \\
(6.9-11.5)\end{array}$ & $\begin{array}{l}U=5.5 \\
P<0.05\end{array}$ \\
\hline $\begin{array}{l}\text { El Casino } \\
\text { (Santa Fe) }\end{array}$ & $19(110)$ & $\begin{array}{l}0.5: 1 \\
X^{2}=1.39 P<0.3\end{array}$ & $\begin{array}{l}0.0086 \pm 0.0019 \\
(0-0.025 \pm 0.0083)\end{array}$ & $\begin{array}{l}\left(\mathrm{U}_{60.50}=1241.5\right. \\
P=0.015)\end{array}$ & $\begin{array}{l}10.4 \\
(5.6-15)\end{array}$ & $\begin{array}{l}12.3 \\
(11.1-15)\end{array}$ & $\begin{array}{l}9.9 \\
(8-13)\end{array}$ & $\begin{array}{l}\mathrm{U}=14 \\
\mathrm{P}<0.04\end{array}$ \\
\hline \multirow{3}{*}{$\begin{array}{l}\text { Barracuda } \\
\text { (Varadero) }\end{array}$} & & $1.1: 1$ & $0.053 \pm 0.0036$ & & 10.3 & 12.5 & 10.4 & $\mathrm{U}=1199$ \\
\hline & $329(306)$ & $\mathrm{X}^{2} 0.36 P<0.7$ & $\begin{array}{l}(0.0206- \\
0.11183 \pm 0.0132)\end{array}$ & $\begin{array}{l}\left(\mathrm{U}_{136.170}=9587\right. \\
P=0.0000)\end{array}$ & $(2.9-17.3)$ & $(6-17.3)$ & $(6-15.8)$ & $P<0.05$ \\
\hline & & $0.5: 1$ & $0.0422 \pm 0.0041$ & & 9.4 & 11.6 & 9.5 & $\mathrm{U}=5254$ \\
\hline $\begin{array}{l}\text { Caleta } \\
\text { (Varadero) }\end{array}$ & $158(170)$ & $X^{2}=11.52 P<0.01$ & $\begin{array}{l}(0.0088- \\
0.094 \pm 0.0218)\end{array}$ & $\begin{array}{l}\left(\mathrm{U}_{119.160}=3387\right. \\
P=0.026)\end{array}$ & $(2.8-18.3)$ & $(6.2-18.3)$ & $(6.2-16.1)$ & \multirow[t]{8}{*}{$P<0.05$} \\
\hline $\begin{array}{l}\text { Cerco } \\
\text { (Caibarién) }\end{array}$ & $10(60)$ & $\begin{array}{l}0.3: 1 \\
X^{2}=1.13 P<0.5\end{array}$ & $\begin{array}{l}0.100 \pm 0.0038 \\
(0-0.045 \pm 0.0138)\end{array}$ & $\begin{array}{l}\left(\mathrm{U}_{20.30}=195\right. \\
P=0.0005)\end{array}$ & $\begin{array}{l}7.9 \\
(3-10.2)\end{array}$ & 8.9 & $\begin{array}{l}9 \\
(7.6-10.2)\end{array}$ & \\
\hline & & & $0.0004 \pm 0.0010$ & & 6.7 & & & \\
\hline $\begin{array}{l}\text { Refugio } \\
\text { (Caibarién) }\end{array}$ & $4(60)$ & & $(0-0.003 \pm 0.0007)$ & & $(4.3-14)$ & & 14 & \\
\hline $\begin{array}{l}\text { Cayo } \\
\text { Francés } \\
\text { (Caibarién) }\end{array}$ & $3(30)$ & & & & $\begin{array}{l}4.3 \\
(3.6-5)\end{array}$ & & & \\
\hline $\begin{array}{l}\text { Marina } \\
\text { Periquillo } \\
\text { (Caibarién) }\end{array}$ & $1(20)$ & & & & & 13.2 & & \\
\hline $\begin{array}{l}\text { Playa } \\
\text { Flamenco } \\
\text { (Cayo } \\
\text { Coco) }\end{array}$ & $4(165)$ & & $(0-0.006 \pm 0.0045)$ & & $\begin{array}{l}5.1 \\
(4-6.2)\end{array}$ & & 6.2 & \\
\hline $\begin{array}{l}\text { Canalizo de } \\
\text { los Perros } \\
\text { (B. de los } \\
\text { Perros) }\end{array}$ & $1(20)$ & & & & & & 6.5 & \\
\hline
\end{tabular}


(Cayo Coco) y las estaciones de Marina Periquillo y Cayo Francés (Caibarién).

Para verificar la distribución normal de los datos se usó la prueba Kolmogorov-Smirnov con el programa Statistica 6.0 (StatSoft, 2002) y para comprobar la homogeneidad de varianza de estos se empleó la prueba de Levene. Como los datos no cumplieron con estos requisitos se emplearon análisis estadísticos no paramétricos.

Los análisis de densidad, talla y reproducción se hicieron para cada especie en general, por estaciones y épocas del año. Para la comparación de medias se aplicaron las pruebas de Kruskal Wallis y U de Mann-Whitney. Como pruebas de comparación múltiple de medias se aplicaron las pruebas Dunn (Zar, 1996), mediante el programa GraphPadInStat y el Posthoc del programa Statistica versión 6.0 (StatSoft, 2002) para pruebas no paramétricas. Se aplicó la prueba Chi Cuadrado (Zar, 1996) para determinar las diferencias entre la proporción sexual esperada 1:1 (Lourie et al. 1999) y la observada por especies y por estaciones. Las diferencias estadísticas fueron consideradas con un nivel de significación del 95\% $(\alpha=0.05)$.

Los sustratos utilizados por las especies $H$. reidi e $H$. erectus fueron identificados hasta el taxón más bajo posible con la ayuda de un especialista. También se analizó la frecuencia de utilización de cada sustrato por ambas especies.

\section{RESULTADOS}

Se encontró un total de 607 individuos, de ellos 569 fueron de la especie $H$. reidi, y 38 de la especie $H$. erectus, por lo que $H$. reidi representó el $93.7 \%$ del total.

Del total de individuos de $H$. reidi, 252 fueron hembras (44.3\%), 215 machos $(37.8 \%)$ y 102 indiferenciados (18\%). La proporción sexual fue de 0.9:1 $\left(X_{\mathrm{c}}^{2}=2.64 P<0.3\right)$, similar a la esperada. El largo total medio (LT) fue de $9.8 \pm 0.2 \mathrm{~cm}$ (rango de 2.4-18.3 $\mathrm{cm})$, y se encontraron diferencias significativas entre las estaciones con $\mathrm{H}(5, \mathrm{~N}=558)=37.4 P<0.01$ (Fig. 2).

Los individuos de mayor talla aparecieron en la estación del Bajo de Santa Ana, como se muestra en la Figura 2, esto puede estar relacionado con la abundancia de alimento en el lugar, debido a que es una zona estuarina cercana a la desembocadura del río del mismo nombre, donde la entrada de nutrientes hace que la productividad biológica sea elevada.

$H$. reidi se encontró en ambientes estuarinos, playas de arena, pastos marinos, manglar sumergido y estructuras artificiales de un canal, en un rango de temperatura entre 19 y $31^{\circ} \mathrm{C}$ $\left(25.1 \pm 0.4^{\circ} \mathrm{C}\right)$ y en un rango de salinidad de 10-39 PSU (33.3 \pm 0.9 PSU).

Las hembras tuvieron un LT medio de $9.9 \pm 0.2$ (rango de 6-16.1 cm), para los machos fue de $12.2 \pm 0.2 \mathrm{~cm}$ (rango de $6-18.3 \mathrm{~cm}$ ) y para los indiferenciados fue de $4.5 \pm 0.1$ (rango de 2.4-5.9 cm). Los machos fueron más grandes que las hembras $U=14566 P<0.01$.

La cantidad de individuos grávidos fue baja con respecto al total de machos (11\%), aunque aparecieron en todos los meses. 


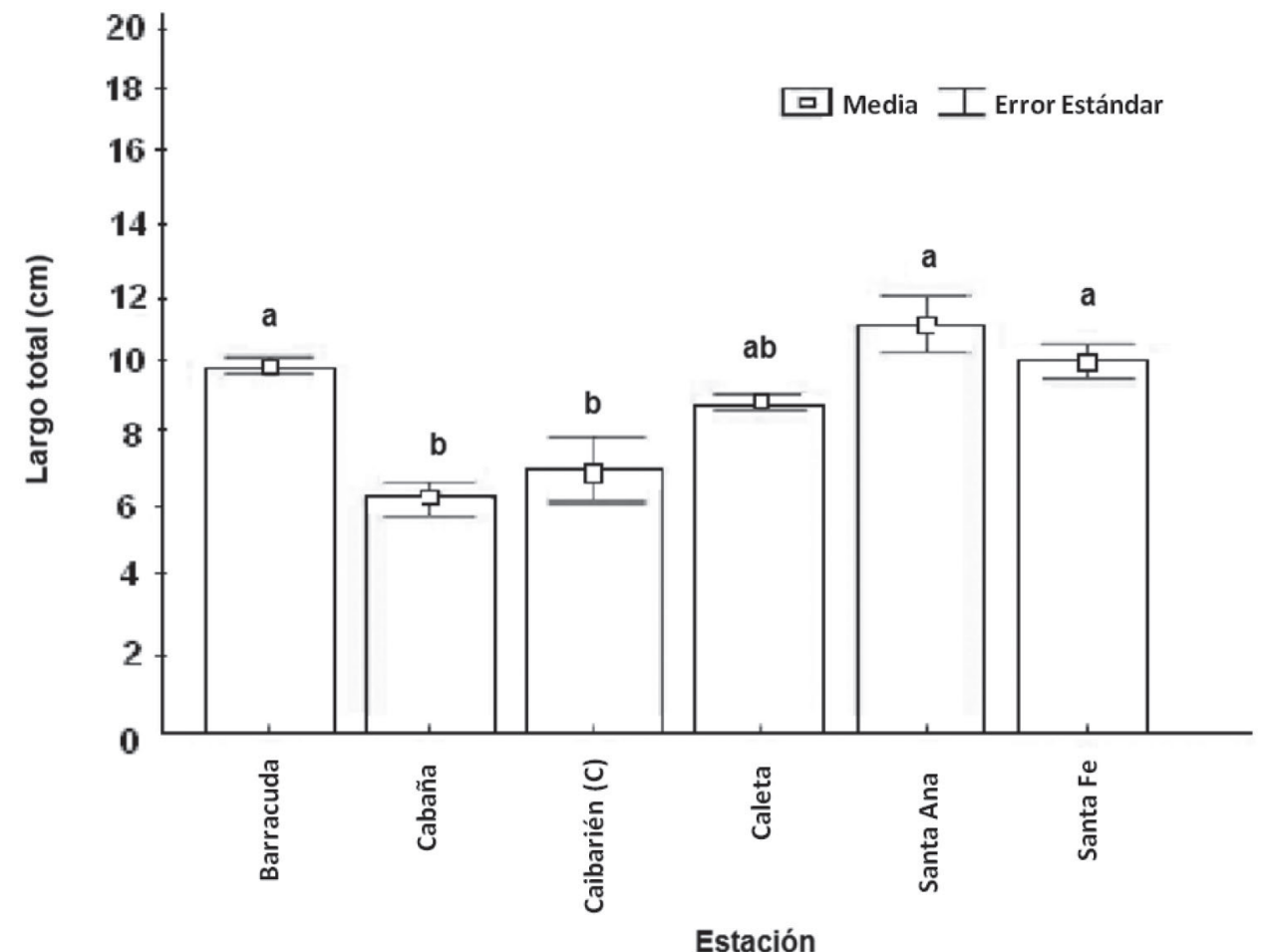

Fig. 2. Largo total de H. reidi por estaciones. (C): Caibarién Cerco. Las letras indican el resultado de la prueba Dunn con significación de 0.05

Fig. 2. Total length of H. reidi per station. (C): Caibarién Cerco. Letters indicate results of the Dunn's test with a 0.05 significance level

No fue posible determinar un pico reproductivo, pero la mayor cantidad de individuos grávidos se encontró en los meses de abril y junio, con una talla media de $12.5 \pm 0.5$ (rango de $6.8-15.4 \mathrm{~cm})$.

En relación con la conducta social de la especie, el $97.5 \%$ de los individuos se reportaron solos. Solamente en la estación de Barracuda aparecieron 7 parejas, de las cuales 4 estaban realizando el cortejo. El tamaño del grupo fue de 2 individuos. Se encontraron peces de colores amarillo, naranja, rojo, negro y pardo, con predominio del color amarillo.
Densidad y utilización de los componentes del hábitat

La densidad media de la especie $H$. reidi fue de $0.0306 \pm 0.0017 \mathrm{ind} /$ $\mathrm{m}^{2}$ y se encontraron diferencias significativas entre las estaciones con $\mathrm{H}(7, \mathrm{~N}=966)=200.5 P<0.01$. Como se observa en la Figura 3, la mayor densidad se obtuvo en la estación de Barracuda $\left(0.053 \pm 0.0036 \mathrm{ind} / \mathrm{m}^{2}\right) \quad y$ la menor en el Refugio (R) Caibarién $\left(0.0004 \pm 0.0010 \mathrm{ind} / \mathrm{m}^{2}\right)$. La densidad en época de lluvia fue similar a la de época de seca ( $\mathrm{U}=102522 P=0.56)$.

$H$. reidi utilizó 15 sustratos de apoyo $(\mathrm{n}=569)$, resultando la 


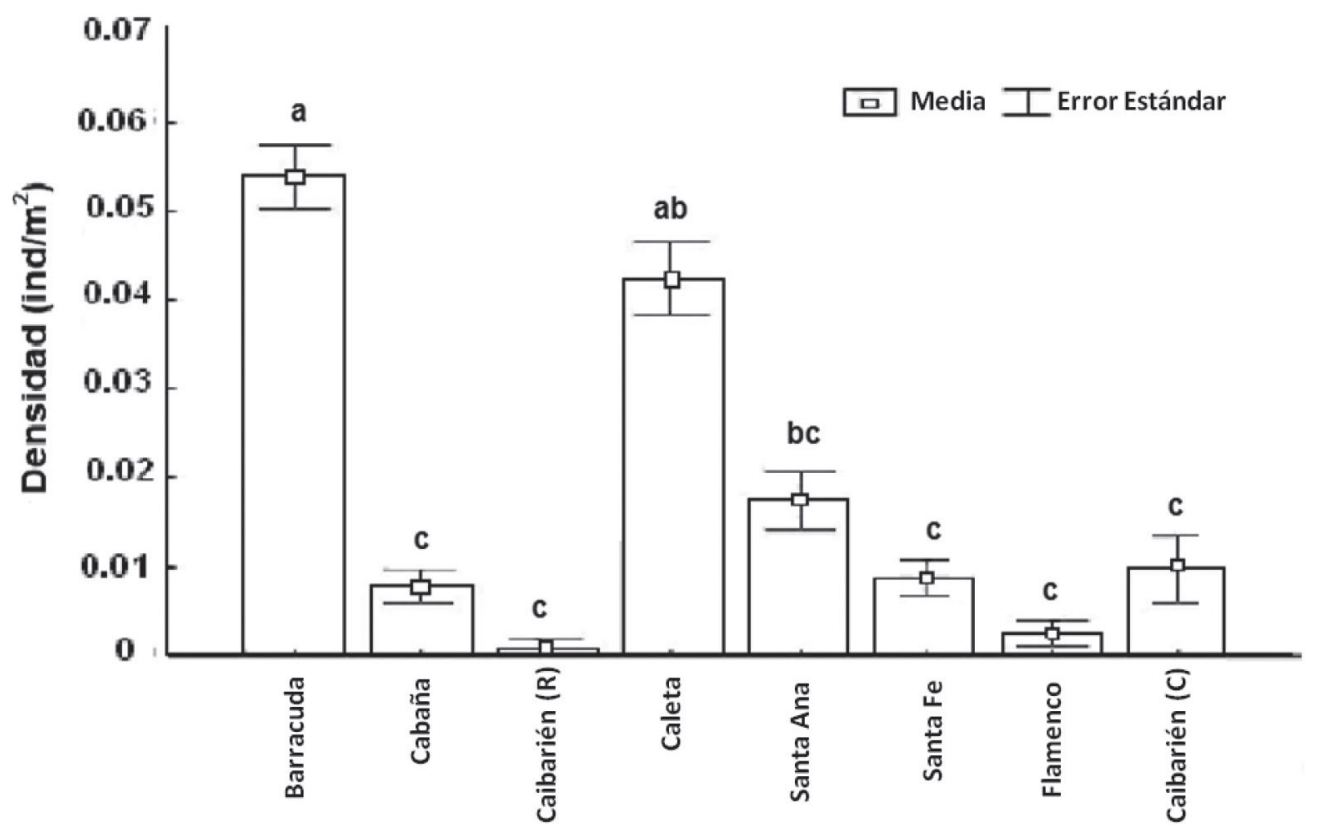

Estación

Fig. 3. Densidad media de H. reidi por estaciones. (R): Caibarién Refugio, (C): Caibarién Cerco. Las letras indican el resultado de la prueba Dunn con significación de 0.05

Fig. 3. Mean density of $H$. reidi per station. (R): Caibarién Refugio, (C): Caibarién Cerco. Letters indicate results of the Dunn's test with a 0.05 significance level

macroalga Caulerpa racemosa, la de mayor frecuencia con $36.5 \%$ del total $(\mathrm{n}=209)$, seguidas por Caulerpa sertularioides (S.G. Gmelin) M.A. Howe (28\%) y Caulerpa pusilla $(12 \%)$. Otros sustratos también utilizados fueron: el poliqueto tubícola Sabellastarte magnifica (8\%), las raíces de mangle rojo Rhizophora mangle (Linnaeus, 1753) (5\%), las esponjas Dysidea etheria y Tedania ignis, las macroalgas Halimeda opuntia (Linnaeus) J. V. Lamouroux, Dictyota sp., y Dictyopteris sp., la fanerógama marina Thalassia testudinium, el bivalvo Isognomon alatus (Gmelin, 1791), ascidias, briozoos y el coral Porites porites.
Los datos poblacionales de la especie $H$. reidi, por estaciones, se presentan en el Cuadro 1. Como se puede observar, en Playa Caleta la proporción sexual fue de 0.5:1 $\left(\mathrm{X}^{2}=11.52 \quad P<0.01\right)$ con un mayor número de hembras que de machos, en el resto de las estaciones no hubo diferencias. Los machos fueron mayores que las hembras en todas las estaciones (Cuadro 1).

La densidad poblacional de la especie por estaciones fue baja, con valores entre $0.0004 \mathrm{ind} / \mathrm{m}^{2}$ y 0.053 $\mathrm{ind} / \mathrm{m}^{2}$ y se encontraron diferencias significativas en la densidad de las épocas de lluvia y seca en las estaciones de Las Cuarenta, El Casino, Refugio 


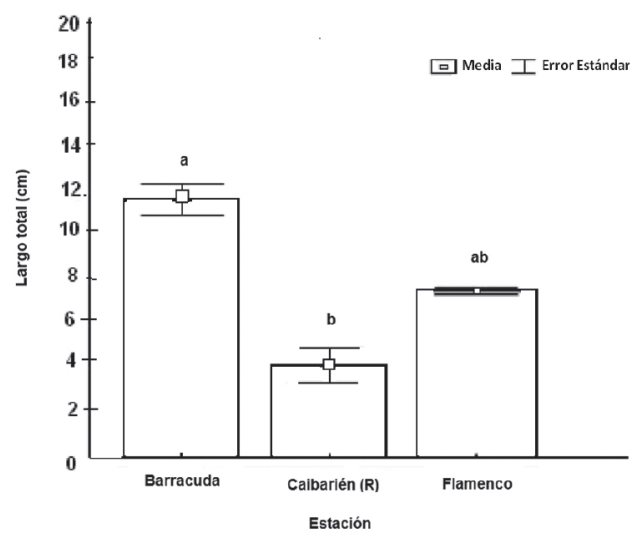

Fig. 4. Largo total de $H$. erectus por estaciones. (R): Caibarién Refugio. Las letras indican el resultado de la prueba Dunn con significación de 0.05

Fig. 4. Total length of $H$. erectus per station. (R): Caibarién Refugio. Letters indicate results of the Dunn's test with a 0.05 significance level

y Cerco, donde la densidad en época de lluvia fue significativamente mayor que la de seca (Cuadro1).

Se encontraron 38 individuos de la especie $H$. erectus, de ellos 26 fueron machos (68\%), 7 hembras $(18 \%)$ y 5 indiferenciados $(13 \%)$. La proporción sexual fue de 3.7:1 $\left(X_{\mathrm{c}}{ }_{\mathrm{c}}=9.82 P<0.01\right)$. El largo total medio (LT) de los individuos fue de $10.6 \pm 0.7 \mathrm{~cm}$ (rango de $2.7-18 \mathrm{~cm}$ ) y se registraron diferencias significativas entre las estaciones con $\mathrm{H}(2, \mathrm{~N}=35)=12.9 P<0.01$, donde los individuos más grandes aparecieron en la estación de Barracuda (Fig. 4).

Esta especie solo se encontró en ambientes netamente marinos, en especial en playas de arena y estructuras artificiales de un canal que tiene salida al mar, en un rango de temperatura

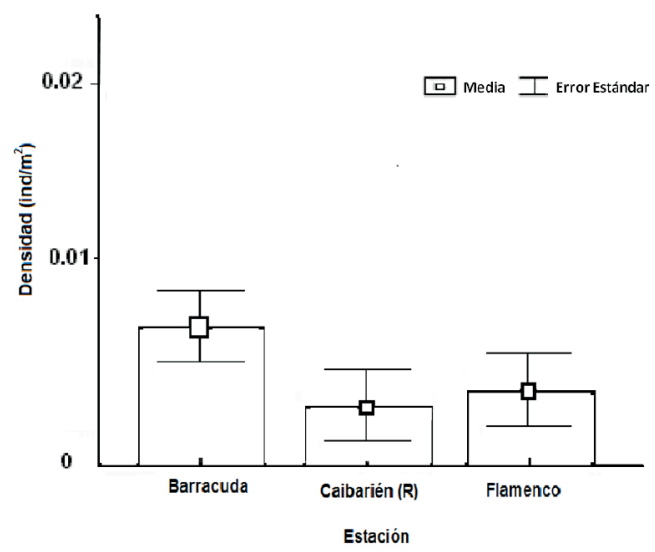

Fig. 5. Densidad media de H. erectus por estaciones. (R): Caibarién Refugio

Fig. 5. Mean density of $H$. erectus per station. (R): Caibarién Refugio

entre 19 y $30^{\circ} \mathrm{C}\left(24 \cdot 6 \cdot 1 \pm 0.7^{\circ} \mathrm{C}\right)$ y en un rango de salinidad entre 14 y 40 PSU (34.7 \pm 1.7 PSU), reafirmando su carácter eurihalino de tolerar amplios rangos de salinidad. Las hembras tuvieron un LT de $8.4 \pm 0.6$ (rango de $6.8-10.5 \mathrm{~cm}$ ), para los machos fue de $12.6 \pm 0.6 \mathrm{~cm}$ (rango de $6.5-18 \mathrm{~cm}$ ) y para los indiferenciados fue de 4.6 \pm 0.5 (rango de 2.7-5.6 cm). Los machos mostraron mayor talla que las hembras $\mathrm{U}=27.5 P<0.01$.

La cantidad de individuos grávidos encontrados en esta especie representó el $38 \%$ del total de machos, con una talla media de $13.4 \pm 0.8$ (rango de 8-18 cm). Solo se registraron en los meses de febrero y octubre del 2008 y enero del 2009, por lo que no se pudo determinar el período reproductivo.

En relación con la conducta social de la especie se encontró que el $100 \%$ de los individuos estaban solos. Predominaron los especímenes de colores pardo oscuro y negro. 
Cuadro 2. Datos poblacionales de H. erectus por estaciones. N: número de individuos, n: número de transectos; M.H: proporción sexual; Media \pm intervalo de confianza (rango); LT: Largo Total y entre paréntesis aparece el rango, H: hembra; M: macho; U: prueba de Mann-Whitney para el LT y para la densidad por época de lluvia y seca

Table 2. Population data for $H$. erectus per station. N: number of individuals, n: number of transects; M: H: sex ratio, mean \pm confidence interval; LT: Total length (range between parentheses), H: female, M: male; U: Mann-Whitney test for TL and for density in rainy and dry seasons

\begin{tabular}{|c|c|c|c|c|c|c|c|c|}
\hline Estación & $\mathbf{N}(n)$ & M:H & $\begin{array}{c}\text { Densidad media } \\
\text { ind } / \mathbf{m}^{2}\end{array}$ & $\begin{array}{l}\text { Prueba U: } \\
\text { Seca/lluvia }\end{array}$ & $\begin{array}{l}\mathrm{LT} \\
(\mathrm{cm})\end{array}$ & $\begin{array}{l}\text { LT } \\
\text { (M) }\end{array}$ & $\begin{array}{l}\text { LT } \\
\text { (H) }\end{array}$ & $\begin{array}{c}\text { Prueba U: } \\
\text { MxH }\end{array}$ \\
\hline El Casino & $1(110)$ & & & & & & 9 & \\
\hline \multirow[t]{2}{*}{ Barracuda } & $26(306)$ & 7:01 & $0.0074 \pm 0.0015$ & $\begin{array}{l}\left(\mathrm{U}_{119.51}=2700.0\right. \\
P=0.05) .\end{array}$ & 11.8 & 13.2 & 9.1 & $\mathrm{U}=8$ \\
\hline & & $\begin{array}{l}\mathrm{X}^{2}=12.04 \\
P<0.01\end{array}$ & $\begin{array}{l}(0.0029- \\
0.0265 \pm 0.0076)\end{array}$ & & $(4.4-18)$ & $(6.6-18)$ & $(6.8-10.5)$ & $P<0.04$ \\
\hline $\begin{array}{l}\text { Playa } \\
\text { Caleta }\end{array}$ & $2(170)$ & & & & $\begin{array}{l}14.2 \\
(12.9-15.5)\end{array}$ & & & \\
\hline \multirow[t]{2}{*}{$\begin{array}{l}\text { Playa } \\
\text { Flamenco }\end{array}$} & $6(165)$ & 1:01 & $0.0040 \pm 0.0018$ & $\begin{array}{l}\left(\mathrm{U}_{45.30}=600\right. \\
P=0.06)\end{array}$ & 7.6 & 7.7 & 7.5 & \\
\hline & & & $(0-0.016 \pm 0.008)$ & & $(7-8.2)$ & $(7.5-8)$ & $(7-8.2)$ & \\
\hline \multirow[t]{2}{*}{ Refugio } & $3(60)$ & & & & 4.1 & & & \\
\hline & & & & & $(2.7-5.5)$ & & & \\
\hline
\end{tabular}

\section{Densidad y utilización de los componentes del hábitat}

La densidad media de la especie $H$. erectus fue de $0.0058 \pm 0.0010 \mathrm{ind} /$ $\mathrm{m}^{2}$. No se encontraron diferencias significativas entre las estaciones con $\mathrm{H}(2, \mathrm{~N}=295)=3.9 P=0.15$, como se observa en la Figura 5. La densidad en época de lluvia fue similar a la de época de seca $(\mathrm{U}=9107.5 P=0.32)$.

H. erectus utilizó 6 sustratos para su anclaje $(n=38)$, mostrando preferencia por las macroalgas: Caulerpa sertularioides (39.5\%), Caulerpa racemosa (21\%), Penicillium sp. (15\%), ascidias $(12 \%)$, briozoos $(7 \%)$ y esponjas.

Los resultados de los datos poblacionales de la especie $H$. erectus por estaciones se presentan en el Cuadro 2. Como se puede observar en Barracuda la proporción sexual fue de 7:1 $\left(\mathrm{X}^{2}=12.04\right.$ $P<0.01)$ con un mayor número de machos que de hembras, en el resto de las estaciones no hubo diferencias. Solo en la estación de Barracuda los machos fueron significativamentemayores quelas hembras $\mathrm{U}=8 P<0.04$ (Cuadro 2). No se encontraron diferencias significativas en la densidad de la especie en época de lluvia y en época de seca en ninguna de las estaciones, aunque los tres individuos que se registraron en Refugio fueron en época de lluvia.

\section{DISCUSIÓN}

En este estudio, la especie $H$. reidi resultó la más abundante en la costa norte 
de las regiones occidental y central de Cuba. Esta especie fue la más abundante también en las costas norte y sur de Brasil según los estudios de Rosa et al. (2002) y Silveira (2011). Sin embargo, en el Golfo de México y las costas orientales de Estados Unidos, $H$. reidi es la menos abundante de las especies presentes (Bruckner et al. 2005).

Según Lourie et al. (2004), H. reidi tiene una amplia distribución vertical en un rango que oscila entre 15 y $55 \mathrm{~m}$ de profundidad; además, es común encontrarla en manglares, pastos marinos, algas, ostras, cnidarios, esponjas, tunicados, estructuras artificiales en estuarios, gorgonáceos y corales pétreos.

En Brasil se ha reportado la presencia de $H$. reidi desde $10 \mathrm{~cm}$ (Rosa et al. 2002) hasta $55 \mathrm{~m}$ de profundidad según Vari (1982). También ha sido confirmada su presencia a pocos metros de la superficie por Rosa et al. (2007) y Oliveria \& Freret-Meurer (2012).

En Cuba, esta especie se ha encontrado entre $50 \mathrm{~cm}$ y $2.5 \mathrm{~m}$ de profundidad (Pastor et al. 2011), esto coincide con los resultados de este estudio.

$H$. reidi apareció en un rango de temperatura y salinidad mayor que el reportado por Freret-Meurer \& Andreata (2008), Mai \& Gonzalo (2012), Ramineli \& Silveira (2013) y menor que el registrado por Silveira (2005) y Pastor et al. (2011).

Se encontró una proporción sexual similar a la esperada (1:1), resultado que coincide con lo reportado por Mai \& Rosa (2009), Pastor et al. (2011) y Ramineli (2012).

En general, los machos de $H$. reidi fueron mayores que las hembras, resul- tados similares obtuvieron Dias (2002), Barros (2005) y Osório (2008), así como Bell et al. (2003) para la especie H. capensis, y Anderson (2012) para $H$. erectus. Según Foster \& Vincent (2004), el dimorfismo sexual en cuanto al largo total es inusual en los caballitos de mar, aunque en algunos individuos las proporciones del cuerpo sí muestran algún grado de dimorfismo, por ejemplo, el macho presenta la cola mayor que las hembras.

La cantidad de individuos grávidos encontrados fue baja y menor que la registrada por Silveira (2005) y Rosa et al. (2007). La presencia de al menos un individuo grávido en todos los meses puede ser un indicador de que la especie se reproduce todo el año como lo reportan Mai \& Rosa (2009) y Mai \& Gonzalo (2012).

En este estudio no se encontró un pico reproductivo para $H$. reidi, aunque la mayor cantidad de machos grávidos apareció en los meses de abril y junio, coincidiendo con el final de la época de seca y principio de la época de lluvia, estos resultados coinciden con Pastor et al. (2011) en un estudio en el período 2004/2005 en las zonas de Las Cuarenta y El Casino.

En Brasil, Silveira (2005) encontró picos reproductivos para esta especie en los meses de junio y octubre, Rosa et al. (2007), en los meses de verano para el hemisferio sur (octubre a febrero), mientras que Mai \& Gonzalo (2012) los reportan en mayo y noviembre, lo cual sugiere que las condiciones ambientales de cada sitio pueden influir en el período reproductivo de la especie. 
La talla de diferenciación sexual, así como el menor macho maduro encontrado en este trabajo, que fueron de 6 y $7.2 \mathrm{~cm}$ de LT, respectivamente, fueron menores que los reportados por Mai \& Rosa (2009) y Mai \& Gonzalo (2012). Es posible que estas diferencias estén relacionadas con que estos autores analizaron un mayor número de individuos por sexo $\mathrm{y}$ clases de largo o con diferencias en el método de muestreo utilizado.

Se encontró un porcentaje alto de individuos solitarios (97.5\%), con un valor superior al reportado por Rosa et al. (2007) y Mai \& Rosa (2009), esto puede deberse a que dichos autores localizaron un mayor número de parejas que las registradas en este estudio.

Perante et al. (2002) plantean que, por lo general, las especies del género Hippocampus son encontradas en parejas relativamente alejadas unas de otras, pero que se mantienen estables debido en parte a la fidelidad que existe entre ellas y al pequeño espacio que utilizan para establecerse. En este estudio solo se localizaron 4 parejas en la estación de Barracuda, en el resto de las estaciones no aparecieron.

Pastor et al. (2011), en un estudio durante el período 2004/2005 en las estaciones de Las Cuarenta y El Casino, encontraron a todos los individuos de $H$. reidi solos, resultados similares reportaron Martin \& Vincent (2005) para H. abdominalis.

En relación con los sustratos más utilizados por esta especie, no coinciden con Mai \& Rosa (2009) y Ramineli \& Silveira (2013), donde las raíces de mangle blanco Laguncularia racemosa, de mangle rojo Rhizophora mangle y el alga parda Sargassum fueron los principales sustratos de apoyo. En este estudio, las macroalgas $\mathrm{y}$, en especial, las del género Caulerpa fueron las más utilizadas para el anclaje de esta especie.

La densidad poblacional en general para $H$. reidi en la costa norte de Cuba fue baja, con valores que oscilaron entre $0.0004 \mathrm{ind} / \mathrm{m}^{2}$ y $0.053 \mathrm{ind} / \mathrm{m}^{2}$ y una media de $0.0306 \pm 0.0017 \mathrm{ind} / \mathrm{m}^{2}$. Esto coincide con lo reportado por Foster \& Vincent (2004), que plantean que los caballitos de mar presentan bajas densidades que pueden estar entre 0.006 y 1.1 individuos $/ \mathrm{m}^{2}$ de acuerdo con la especie.

Al comparar esta densidad con la reportada por otros autores, encontramos que fue mayor que la registrada por Arcos-Pulido \& GómezPrieto (2004), Rosa et al. (2007), Osório (2008) y Pastor et al. (2011) y menor que la hallada por Freret-Meurer \& Andreata (2008) y Mai \& Rosa (2009) en diferentes zonas de Brasil.

El uso de estructuras artificiales como sustratos de anclaje o de apoyo se ha observado en algunas especies de Hippocampus (Foster \& Vincent, 2004), y también se ha reportado para $H$. reidi por Dias \& Rosa (2003) y Oliveira (2007), lo que sugiere una relativa plasticidad de esta especie en relación con modificaciones antrópicas en los ecosistemas costeros, aunque los hace más vulnerables a la explotación (Mai \& Rosa, 2009).

En este estudio, las densidades más altas se obtuvieron en las estructuras 
artificiales (muelle de hormigón y de madera) de las estaciones de Barracuda y Caleta, que están dentro de un canal con salida al mar $(0.053 \pm 0.0036$ y $0.042 \pm 0.0041 \mathrm{ind} / \mathrm{m}^{2}$ ), con valores superiores al encontrado por Pastor et al. (2011) en estructuras artificiales de El Casino, Santa Fe $\left(0.0037 \mathrm{ind} / \mathrm{m}^{2}\right)$ en el período 2004/2005, y menor que la registrada por Dias \& Rosa (2003) en un muelle de madera $\left(0.51 \mathrm{ind} / \mathrm{m}^{2}\right)$ en Río Grande al noreste de Brasil.

Las estaciones de Barracuda y Caleta están próximas a la Bahía de Cárdenas en la provincia de Matanzas, por lo que el constante aporte de nutrientes provenientes de ella y de los desagües de albañales que existen en el lugar, han provocado niveles de eutrofización que han estimulado el desarrollo de abundantes especies de macroalgas, así como especies sésiles filtradoras como esponjas, bivalvos, poliquetos tubícolas, ascidias, briozoos, entre otros organismos que se encuentran asociados a las paredes y pilotes de los muelles y que brindan amplias oportunidades para el anclaje, el camuflaje y la alimentación de los caballitos. Las aguas eutrofizadas han favorecido también la presencia de caballitos de mar en el mar Piccolo di Taranto, en Italia, debido al incremento de las poblaciones de crustáceos, presas potenciales de estos peces, según sugieren Gristina et al. (2015).

Rosa et al. (2007) encontraron que la disponibilidad de hábitats parece influir en la abundancia y distribución espacial de la especie $H$. reidi, sugerida por el uso de briozoos y macroalgas abundantes en las estaciones de la costa sur de Brasil, mientras que las raíces de mangle lo fueron en las estaciones de la costa norte. Según Aylesworth et al. (2015), en un estudio sobre preferencia dehábitats de esta misma especie, fueron precisamente la abundancia de sitios de anclaje, así como la poca profundidad y las temperaturas cálidas en el agua, las variables más importantes para predecir la presencia de los hipocampos en los estuarios tropicales.

Es posible que la gran diversidad de macroalgas e invertebrados sésiles presentes en las estaciones de Barracuda y Caleta también favorezcan los patrones de coloración de los individuos, donde se encontraron especímenes amarillos, naranjas, rojos, negros, marrones, pardos claros y oscuros a diferencia del resto de las estaciones, donde solo aparecieron individuos pardos claros, oscuros y negros. En ambas estaciones predominaron los colores amarillos y naranjas.

La densidad encontrada en la estación de Las Cuarenta fue menor que la obtenida por Pastor et al. (2011) en la misma zona entre los meses de abril del 2004 y abril del 2005 (0.010 ind/ $\mathrm{m}^{2}$ ), lo que indica una disminución de la cantidad de especímenes en el área. Es posible que el incremento de pescadores furtivos, la utilización de redes de arrastre en zonas cercanas y el buceo de turistas nacionales en esta zona, sean las causas principales de esta disminución.

En el estuario del río de Santa Ana, la densidad fue menor que la obtenida para los ríos Maracaipe, Camurupin/Cardoso y Timonha/Ubatuba (Silveira, 2005; Mai \& Rosa, 2009) y mayor que la encontrada por 
Dias \& Rosa (2003) en Río Grande (0.006 $\mathrm{ind} / \mathrm{m}^{2}$ ) y Osório (2008) en los estuarios de los ríos de Pacoti y Malcozinhado (0.008 ind $/ \mathrm{m}^{2}$ ) en Brasil.

La especie $H$. erectus es más frecuente encontrarla en aguas profundas donde la vegetación es abundante (Teixeira \& Musick, 2001). Se ha reportado a una profundidad máxima de $73 \mathrm{~m}$, principalmente en pastos marinos, asociados a esponjas y macroalgas flotantes (Sargassum), según Lourie et al. (2004).

En Brasil, H. erectus generalmente se captura como fauna acompañante en las pesquerías del camarón blanco Litopenaeus schmitt, reforzando el criterio de que es más frecuente a mayor profundidad, aunque también se ha encontrado en aguas poco profundas en la costa sur, a pocos metros de la superficie, asociados a macroalgas y briozoos para su anclaje (Rosa et al. 2011). En este estudio, la especie se registró entre 1 y $2 \mathrm{~m}$ de profundidad, es posible que el método de muestreo utilizado sea la causa de la poca cantidad observada de individuos.

$H$. erectus apareció en un rango de temperatura menor que el reportado por Teixeira \& Musick (2001) y en un rango de salinidad mayor, esto reafirma el criterio de estos autores de que es una especie eurihalina que tolera amplios rangos de salinidad.

Los machos de $H$. erectus presentaron tallas mayores que las hembras, esto coincide con Anderson (2012), que en un estudio sobre dimorfismo sexual en adultos de esta especie, encontró que los machos tienen mayor talla y colas más largas que las hembras.

Se encontró una proporción sexual significativamente diferente a la esperada, resultados similares obtuvieron Teixeira \& Musick (2001) en la Florida, Perante et al. (1998), Bell et al. (2003) y Martin \& Vincent (2005) en otras especies de caballitos. Pastor et al. (2011) no pudieron determinar esta proporción por la ausencia de machos en las zonas de Las Cuarenta y El Casino durante el período muestreado.

La cantidad de individuos grávidos fue baja con respecto al total de machos (38\%). Al solo aparecer en tres meses, no se pudo determinar la época reproductiva para la especie $H$. erectus, a diferencia de Teixeira \& Musick (2001) que reportan picos reproductivos de mayo a octubre y Rosa et al. (2011) los registraron de enero a marzo.

El $100 \%$ de los individuos de $H$. erectus se encontraron solos, esto coincide con Claro et al. (2001) que plantean que esta especie es más comúnmente registrada sola que formando grupos.

En relación con los sustratos más utilizados por $H$. erectus en este estudio, coinciden con Rosa et al. (2011) y difieren con lo reportado por Pastor et al. (2011), donde las raíces de mangle rojo Rhizophora mangle fue el único sustrato utilizado por esta especie en la estación de Las Cuarenta.

La densidad poblacional, en general, para $H$. erectus en la costa norte de Cuba fue baja, con valores que oscilaron entre $0.004 \mathrm{ind} / \mathrm{m}^{2}$ y 0.0074 
ind $/ \mathrm{m}^{2}$ y una media de $0.0058 \pm 0.0010$ $\mathrm{ind} / \mathrm{m}^{2}$, aunque fue mayor que la registrada por Pastor et al. (2011) de $0.0012 \mathrm{ind} / \mathrm{m}^{2}$ en dos zonas de la costa norte de Cuba y similar a la reportada por Rosa et al. (2011) de 0.005-0.035 ind $/ \mathrm{m}^{2}$ en áreas de Brasil.

Durante este estudio, la especie $H$. erectus no apareció en los tres años de muestreo en la zona de Las Cuarenta (Bahía de Cabaña), lugar donde anteriormente se había reportado su presencia por Pastor et al. (2011), aunque en muy bajos valores de densidad.

\section{CONCLUSIONES}

La densidad poblacional de las especies $H$. reidi e $H$. erectus en general y por estaciones fue baja en la costa norte de las regiones occidental y central de Cuba. La especie $H$. reidi fue la más abundante y la de mayor distribución. La mayor densidad de caballitos se encontró en las estructuras artificiales de las estaciones de Barracuda y Caleta. No se resgistraron diferencias entre las épocas de lluvia y seca en ninguna de las dos especies. Los machos de $H$. reidi y de $H$. erectus fueron mayores que las hembras. La especie $H$. reidi se diferencia sexualmente a una talla menor que la reportada en la literatura. Para $H$. reidi se observó una proporción sexual similar a la esperada de 1:1, mientras que en la especie $H$. erectus la proporción sexual encontrada fue diferente a la esperada. Las macroalgas del género Caulerpa constituyeron los principales sustratos de apoyo para estas especies de caballito de mar.

\section{AGRADECIMIENTOS}

Agradecemos a todos los compañeros del grupo de acuariología del Acuario Nacional de Cuba por su valiosa ayuda en los diferentes muestreos que se realizaron para este trabajo y a los evaluadores por los valiosos aportes.

\section{BIBLIOGRAFÍA}

Anderson, A. P. (2012). Sexual Dimorphism in Morphometry and Allometry of the Adult Lined Seahorse, Hippocampus erectus. Copeia, 3, 389-393. https:// doi.org/10.1643/CG-11-003

Arcos-Pulido, M. \& Gómez-Prieto, C. (2004). Valoración de la población de Hippocampus reidi en la región de Santa Marta. Recuperado en diciembre 10, 2004, disponible en http://www.sur.iucn. org/listaroja/documentos/Hippocampus Aylesworth, L. A., Xavier, J. H., Oliveira, T. P., Tenorio, G. D., Diniz, A. F. \& Rosa, I. L. (2015). Regional-scale patterns of habitat preference for the seahorse Hippocampus reidi in the tropical estuarine environmental. Aquat. Ecol., 49, 499-512. https://doi. org/10.1007/s10452-015-9542-3

Barros, A. T. (2005). Taxonomia e análise da estrutura populacional comercializada de cavalos-marinhos (Syngnathidae: Teleostei: Hippocampus) no Brasil. Dissertation Unpublished, Universidade Federal da Paraíba, João Pessoa, Brasil.

Bell, E. M., Lockyear, J. F., McPherson, J. M., Marsden, A. D. \& Vincent, A. C. (2003). First field studies of an Endangered South African seahorse, Hippocampus capensis. Env. Biol. Fish., 67, 35-46. https://doi. org/10.1023/A:1024440717162 
Bruckner, A. W., Field, J. D. \& Daves, N. (Eds.). (2005). The Proceedings of the International Workshop on CITES Implementation for Seahorse Conservation and Trade. NOAA Technical Memorandum. Maryland, EE. UU.: NOAA.

CITES. (2004). Convención sobre el Comercio Internacional de Especies Amenazadas de Fauna y Flora Silvestres. Recuperado en marzo 18, 2004, disponible en www.cites.org/common/com/AC/20

Claro, R., Lindeman, K. C. \& Parenti, L. R. (2001). The Marine Ichthyofauna of Cuba. In R. Claro, K. C. Lindeman \& L. R. Parenti (Eds.), Ecology of the Marine Fishes of Cuba (pp. 21-32). Washington, D.C., EE. UU.: Smithsonian Institution.

Curtis, J., Moreau, M. A., Marsden, D., Bell, E., Martin-Smith, K., Samoilys, M. \& Vincent, A. (2004). Underwater visual census for seahorse population assessments. Vancouver, B.C., Canada: Fisheries Centre.

de la Nuez, D., Pastor, L., Pérez-Angulo, A., Piloto, Y. \& Corrada, R. I. (2016). Fidelidad al sitio y rango de hogar del caballito de mar narizón Hippocampus reidi (Teleostei: Syngnathidae) en la dársena de Varadero, noroeste de Cuba. Rev. Mar. Cost., 8(1), 95-112. https://doi.org/10.15359/revmar.8-1.7

Dias, T. L. P. (2002). Ecologia populacional de Hippocampus reidi Ginsburg, 1933 (Teleostei: Syngnathidae) no Estado do Rio Grande do Norte, Brasil. Dissertation Unpublished, Universidade Federal da Paraíba, João Pessoa, Brasil.

Dias, T. L. P. \& Rosa, I. M. L. (2003). Habitat preferences of a seahorse species, Hippocampus reidi (Teleostei: Syngnathidae) in Brazil. J. Ichthyol. Aquat. Biol., 6, 165-176.
Foster, S. J. \& Vincent, A. C. J. (2004). Life history and ecology of seahorses: implications for conservation and management.J.Fish. Biol., 65, 1-61.https://doi. org/10.1111/j.0022-1112.2004.00429.x

Freret-Meurer, N. V. \& Andreata, J. V. (2008). Field Studies of a Brazilian Seahorse Population, Hippocampus reidi Ginsburg, 1933. Braz. Arch. Biol. Techn., 51(4), 743-751.

Gaceta Oficial de la República de Cuba (2011). Resolución No. 160/2011 sobre Regulaciones para el control y la protección de especies de especial significación para la diversidad biológica en Cuba. MINJUS. No 26. La Habana, Cuba.

Gristina, M., Cardone, F., Carlucci, R., Castellano, L., Passarelli, S. \& Corriero, G. (2015). Abundance, distribution and habitat preference of Hippocampus guttulatus and Hippocampus hippocampus in a semi-enclosed central Mediterranean marine area. Mar. Ecol., 36, 57-66. https://doi.org/10.1111/ maec. 12116

Harasti, D. (2016). Declining seahorse populations linked to loss of essential marine habitats. Mar. Ecol. Prog. Ser., 546, 173-181. https://doi.org/10.3354/ meps11619

Loh, T.-L., Tewfik, A., Aylesworth, L. \& Phoonsawat, R. (2016). Species in wildlife trade: socio-economic factors influence seahorse relative abundance in Thailand. Biol. Conserv., 201, 301-308. https://doi.org/10.1016/j. biocon.2016.07.022

Lourie, S. A., Vincent, A. C. J. \& Hall, H. J. (1999). Seahorses: an identification guide to the world's species and their conservation. London, U. K.: Project Seahorse. 
Lourie, S. A., Foster, S. J., Cooper, E. W. T. \& Vincent, A. C. J. (2004). A Guide to the Identification of Seahorses. Washington, D. C., EE. UU.: University of British Columbia and World Wildlife Fund.

Lourie, S. A., Pollom, R. A. \& Foster, S. J. (2016). A global revision of the Seahorses Hippocampus Rafinesque 1810 (Actinopterygii: Syngnathiformes): Taxonomy and biogeography with recommendations for further research. Zootaxa, 4146(1), 1-66. https:// doi.org/10.11646/zootaxa.4146.1.1

Mai, A. C. G. \& Rosa, I. M. L. (2009). Aspectos ecológicos do cavalo-marinho Hippocampus reidi no estuário Camurupim/Cardoso, Piauí, Brasil, fornecendo subsídios para acriação de uma Área de Proteção Integral. Biota Neotrop., 9(3), 85-91. https://doi.org/10.1590/ S1676-06032009000300007

Mai, A. G. \& Gonzalo, V. (2012). Population dynamics and reproduction of wild longsnout seahorse Hippocampus reidi. J. Mar. Biol. Assoc. UK, 92(2), 421-427. https://doi.org/10.1017/ S0025315411001494

Martin, K. M. \& Vincent, A. C. J (2005). Seahorse declines in the Derwent estuary, Tasmania in the absence of fishing pressure. Biol. Conserv., 123(4), 533-545. https://doi.org/10.1016/j. biocon.2005.01.003

Oliveira, T. P. R. (2007). Ecologia populacional de Hippocampus reidi (Teleostei: Syngnathidae) em does estuários do estado de Paernambuco, Brasil. Dissertacao de Mestrado Unpublised, Universidade Federal de Paraíba, João Pessoa.

Oliveira, V. \& Freret-Meurer, N. (2012). Distribuição vertical do cavalo-marinho Hippocampus reidi Ginsburg, 1933 na região de Arraial do Cabo, Rio de Janeiro, Brasil. Biotemas, 25(2), 59-66.
Osório, F. M. (2008). Estudo populacional do cavalo-marinho Hippocampus reidi Ginsburg, 1933 (Teleostei: Syngnathidae) em dois estuarios cearenses. Dissertacao de Mestrado Unpublised, Universidade Federal do Ceará, Fortaleza, Brasil.

Pastor, L., Piloto, Y., Corrada, R. \& Chevalier, P. (2011). Estudio de las poblaciones de caballitos de mar en dos zonas de la costa norte de La Habana y Pinar del Río. Rev. Mar. Cost., 3, 171-181.

Perante, N. C., Vincent, A. C. J. \& Pajaro, M. G. (1998). Demographics of the seahorse Hippocampus comes in the central Philippines. In B. Morton (Ed.), Proceedings of the 3rd International Conference on the Marine Biology of the South China Sea (pp. 439-448). Hong Kong, China: University Press.

Perante, N. C., Pajaro, M. G., Meewig, J. J. \& Vincent, A. C. J. (2002). Biology of a seahorse species Hippocampus comes in the central Philippines. J. Fish. Biol., 60, 821-837. https:// doi.org/10.1111/j.1095-8649.2002. tb02412.x

Ramineli, S. M. (2012). Aqui nãotem, não: uma pesquisa socioambiental para a conservação de cavalos-marinhos (Syngnathidae: Hippocampus) emParaty/RJ. Dissertação de Mestrado Unpublised, UFF. Niterói, Brasil.

Ramineli, S. M. \& Silveira, R. B. (2013, septiembre). A população de cavalosmarinhos (Hippocampus reidi) no costãorochoso da praia do cruzeiro, saco do Mamanguá, Paraty. XI Congresso de Ecologia do Brasil, Porto Seguro, Brasil. Rosa, I. L., Dias, T. L. \& Baum, J. K. (2002). Threatened fishes of the world: Hippocampus reidi Ginsburg, 1933 (Syngnathidae). Environ. 
Bio. Fish., 64(4), 378. https://doi. org/10.1023/A:1016152528847

Rosa, I. L., Oliveira, T. P. R., Castro, A. L. C., Moraes, L. E., Xavier, J. H. A., Nottingham, M. C. \& Monteiro-Neto, C. (2007). Population characteristics, space use and habitat associations of the seahorse Hippocampus reidi (Teleostei: Syngnathidae). Neot. Icht., 5(3), 405-414. https://doi.org/10.1590/ s1679-62252007000300020

Rosa, I. L., Oliveira, T. P. R., Osório, F. M., Moraes, L. E., Castro, A. L. C., Barros, G. M. L. \& Alves, R. R. N. (2011). Fisheries and trade of seahorses in Brazil: historical perspective, current trends, and future directions. Biodivers. Conserv., 20(9), 19511971. 10.1007/s10531-011-0068-2

Silveira, R. B. (2005). Dinamica populacional do cavalo-marinho Hippocampus reidi no manguezal de Maracal'pe, Ipojuca, Pernambuco, Brasil. Tesis doctoral no publicada, Pontificia Universidade Católica do Rio Grande, Porto Alegre, Brasil.

Silveira, R. B. (2011). Registros de cavalos-marinhos (Syngnathidae: Hippocampus) ao longo da costa Brasileira. Laboratório de Aqüicultura Marinha (LABAQUAC)-Projeto Hippocampus. Rua da Esperança, 700, Porto de
Galinhas, Ipojuca, PE. Brasil. Oecol. Aust., 15(2), 316-325. https://doi. org/10.4257/oeco.2011.1502.09

StatSoft, Inc. (2002). STATISTICA 6.0 for Windows. Tulsa, Oklahoma, EE. UU.: StatSoft.

Teixeira, R. L. \& Musick, J. A. (2001). Reproduction and food habits of the lined seahorse, Hippocampus erectus (Teleostei: Syngnathidae) of Chesapeake Bay, Virginia. J. Braz. Biol., 61(1), 79-90. https://doi.org/10.1590/ s0034-71082001000100011

UICN. (2009). Red List of Threatened Species. Recuperado en noviembre 20, 2009, disponible en http://www.iucnredlist.org/apps/redlist.html

Vari, R. P. (1982). Fishes of the Western North Atlantic, subfamily Hippocampinae. The seahorses. Mar. Res. Mem., New Haven, 1, 173-189.

Vincent, A. (1995). Trade in seahorses for traditional Chinese medicines, aquarium fishes and curios. Traf. Bullt., 15(3), 125-129.

Yasué, M., Nellas, A., Panes, H. \& Vincent, A. C. (2015). Monitoring landed seahorse catch in a changing policy environment. Endang. Species Res., 27, 95-111. https://doi.org/10.3354/esr00643

Zar, J. H. (1996). Biostatilstical Analysis. New Jersey, EE. UU.: Prentice Hall Inc. 